\title{
Stent-Jack Technique for Ruptured Vertebral Artery Dissecting Aneurysm Involving the Origin of Posterior Inferior Cerebellar Artery
}

\author{
Toshitsugu Terakado, $\mathrm{MD}^{1}$, Yasunobu Nakai, MD, $\mathrm{PhD}^{1}$, Go Ikeda, MD, PhD ${ }^{1}$, Kazuaki Tsukada, $\mathrm{MD}^{1}$, \\ Sho Hanai, $\mathrm{MD}^{1}$, Kazuki Akutagawa, $\mathrm{MD}^{1}$, Haruki Igarashi, $\mathrm{MD}^{1}$, Takahiro Konishi, $\mathrm{MD}^{2}$, \\ Masanari Shiigai, $\mathrm{MD}^{2}$, Kazuya Uemura, $\mathrm{MD}, \mathrm{PhD}^{1}$ \\ ${ }^{1}$ Department of Neurosurgery, Tsukuba Medical Center Hospital, Tsukuba, Japan \\ ${ }^{2}$ Department of Radiology, Tsukuba Medical Center Hospital, Tsukuba, Japan
}

\begin{abstract}
We herein report a case of a ruptured vertebral artery dissecting aneurysm involving the origin of the posterior inferior cerebellar artery that was treated using the stent-jack technique. After parent artery occlusion of the distal vertebral artery, stenting of the posterior inferior cerebellar artery was performed. Further coiling was needed because distal vertebral artery recanalization occurred due to transformation of the coil mass. The stent-jack technique for a ruptured vertebral artery dissecting aneurysm involving the origin of the posterior inferior cerebellar artery is effective; however, careful attention to recanalization after stenting is needed due to transformation of the coil mass.
\end{abstract}

Key Words: Dissecting aneurysm; Vertebral artery; Subarachnoid hemorrhage

\section{INTRODUCTION}

The stent-jack technique is performed for stent-assisted coiling (SAC) of wideneck intracranial aneurysms. The coil mass is pushed back into the aneurysm sac protruding into the main artery via the deployment of a self-expandable stent. 'We herein performed parent artery occlusion (PAO) of the vertebral artery (VA) to treat a vertebral artery dissecting aneurysm (VADA) involving the origin of the posterior inferior cerebellar artery (PICA) using the stentjack technique such that PICA flow was preserved. We describe the treatment procedure and provide technical advice.

\section{CASE REPORT}

A 45-year-old man presented to our hospital with impaired consciousness, a Glasgow Coma Scale of 3, pinpoint pupils, and no breathing difficulties. Brain computed tomography $(\mathrm{CT})$ revealed a subarachnoid hemorrhage (World Federation of Neurosurgical Societies grade 5, Fisher group 3). CT angiography showed a right VADA. Digital subtraction angiography (DSA) showed that the right PICA arose from the dilated part of the $V A$, while the anterior spinal artery originated from the distal portion of the right VA (Fig. 1A). The left VA was of a sufficient size and not hypoplastic. The

\section{Correspondence to:}

Toshitsugu Terakado, MD

Department of Neurosurgery, Tsukuba Medical Center Hospital, 1-3-1

Amakubo, Tsukuba 305-8558, Ibaraki, Japan

Tel: +81-29-851-3511

Fax: +81-29-858-2773

E-mail: toshi_0211jp@yahoo.co.jp

Received: November 26, 2019

Revised: March 12, 2020

Accepted: March 15, 2020

Copyright $\odot 2020$ Korean Society of Interventional Neuroradiology

This is an Open Access article distributed under the terms of the Creative Commons Attribution Non-Commercial License (http://creativecommons.org/licenses/by-nc/4.0) which permits unrestricted non-commercial use, distribution, and reproduction in any medium, provided the original work is properly cited.

pISSN 2093-9043 eISSN 2233-6273 
maximum diameter of the dissection was $5.3 \mathrm{~mm}$, the proximal VA diameter was $2.6 \mathrm{~mm}$, and the PICA diameter was 1.6 $\mathrm{mm}$. We attempted PAO of the VA with PICA stenting and flow preservation. Aspirin at $300 \mathrm{mg}$ and clopidogrel at 300 mg were administered before the treatment. The procedure was performed under general anesthesia. A 4-Fr sheath was inserted into the left femoral artery and a 4-Fr catheter was placed into the left VA. A 6-Fr sheath was inserted into the right femoral artery. A 6-Fr Envoy MPD (Johnson \& Johnson, Miami, FL, USA) was placed into the $\mathrm{V} 1$ segment of the right VA, and a 2.8-Fr/2.3-Fr Plowler Select Plus (Johnson \& Johnson) was introduced into the right PICA. A 1.7-Fr Headway (TERUMO Corporation, Tokyo, Japan) was initially inserted into the dilated part of the VA, and PAO of the distal part of the VA to the PICA bifurcation was performed using Axium Prime coils (Medtronic, Minneapolis, MN, USA). The entire length of the VADA involving the origin of PICA was sparsely embolized (Fig. 1B). In this phase, antegrade blood flow in the VA disappeared (Fig. 1C), and an Enterprise VRD $4 \times 30$ mm (Johnson \& Johnson) was deployed from the PICA to the proximal VA (Fig. 1D). DSA after deployment of the stent showed the recanalization of antegrade blood flow in the VA by the coil mass (Fig. 1E). A Headway was placed into the distal portion of the VA using the transcell technique, and coils were added until PAO was complete (Fig. 1F, G). Aspirin at $100 \mathrm{mg}$ and clopidogrel at $75 \mathrm{mg}$ were administered from the next day of treatment. Magnetic resonance imaging after treatment showed no ischemic events, and DSA 1 week after treatment showed the disappearance of the dissection (Fig. $1 \mathrm{H})$. The patient had no new neurological deficit and was discharged to the rehabilitation hospital with modified Rankin Scale 3. Patient consent was obtained for this case report.

\section{DISCUSSION}

A ruptured VADA is associated with a high mortality rate of $46 \%$ to $67 \%$, and the rerupture rate in the acute phase is $50 \%$ to $70 \%$. Rerupture occurs within 24 hours of disease onset. ${ }^{2,3}$ Occlusion of the entire length of the dissection is necessary to prevent rerupture. ${ }^{4,5} \mathrm{~A}$ ruptured VADA involving the origin of PICA has a less favorable outcome than the PICA non-in-
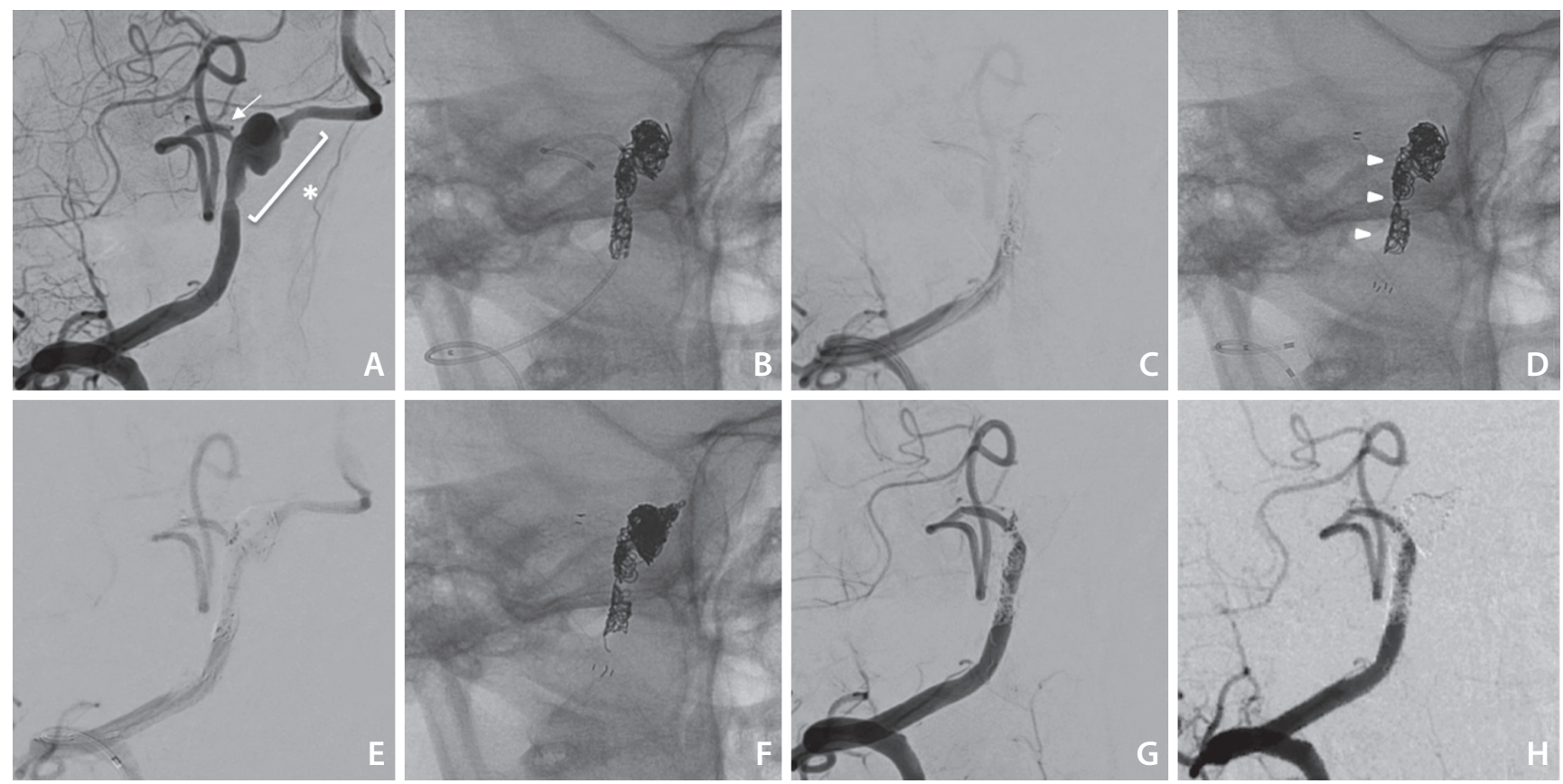

Fig. 1. Treatment course. (A) Posterior inferior cerebellar artery (PICA) (arrow) branches from the middle of the vertebral artery dissecting aneurysm (VADA). The entire length of the dissection (asterisk) needs to be embolized. (B) The distal vertebral artery (VA) from the PICA was densely embolized, while the proximal VA involving the PICA was sparsely embolized. (C) Antegrade VA blood flow disappeared after coiling. (D) The coil shape was changed (arrowheads) by the deployment of Enterprise VRD 4×30 mm. (E) Recanalization of antegrade VA blood flow just after stenting. (F) The coil shape after treatment. The distal VA was densely embolized, while the proximal VA was sparsely embolized and the stent was deployed from the PICA to VA. (G) VADA disappeared, and PICA flow was preserved as shown by digital subtraction angiography just after the treatment. (H) VADA disappeared 1 week after treatment. 
volvement type, and the complication rate associated with this treatment is higher ${ }^{6}$ due to the associated difficulties, as the PICA must be preserved and the dissection occluded.

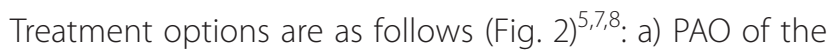
entire length of the VA and occipital artery-PICA bypass, b) proximal VA occlusion to decrease blood flow to the dissection, c) PAO with PICA stenting, and d) stenting to the VA (with coiling of the dissection). Regarding their specifics, a) is a radical, but challenging procedure, b) is relatively easy and associated with a low incidence of rerupture, ${ }^{9}$ but is not radical as the dissection remains, and c) and d) have been performed recently for a ruptured VADA involving the origin of PICA in light of developments in intracranial stents.

The strength of stenting to the VA with coiling of the dissection is that antegrade VA blood flow remains. Therefore, the treatment may be performed on the dominant side of the VADA. The weakness of this technique is the risk of recurrence or rebleeding. Chen et al. ${ }^{10}$ reported 2 cases of recurrence (5.4\%) and 2 of rebleeding (5.4\%) among 37 cases treated by stenting to the VA with coiling of the dissection for a VADA involving the origin of PICA. A PICA territory thromboembolic event may then occur because the stent is implanted to cover the origin of the PICA. The strength of the stent-jack technique is in its potential to reduce recurrence or rebleeding because the coil fills the entire part of the
VADA. The weaknesses of this technique are that it cannot be performed on the dominant side of the VADA and it carries an increased risk of a thromboembolic event in the PICA territory due to the stent being implanted into the PICA. Five cases of VADA involving the origin of PICA with PICA stenting were performed, with occluded PICA occurring in the acute phase in 1 case. ${ }^{8,11}$ However, dual antiplatelet therapy (DAPT) loading was administered after the procedure in these cases. A previous study showed that the rate of a thromboembolic event with DAPT loading after stenting was 4.54-fold higher in ruptured than in unruptured cases, and was only 1.34fold higher in ruptured than in unruptured cases with DAPT loading before stenting. ${ }^{11}$ Therefore, DAPT loading needs to be administered before PICA stenting. Another reason for thromboembolic events is that the PICA is a narrow vessel that is associated with a high risk of occlusion with stenting. However, previous studies reported safe and successful Enterprise placement in vessel diameters less than $2 \mathrm{~mm}$. $^{12,13}$ In 2 cases, thrombus formation occurred but dissolved after intra-arterial administration of a glycoprotein IIb/Illa inhibitor. In any case, adequate antiplatelet therapy is very important.

The limitations of the endovascular treatment of VADA involving the origin of PICA include the need for tight packing even though the dissection is unstable and vulnerable to rebleeding in addition to the preservation of the PICA to

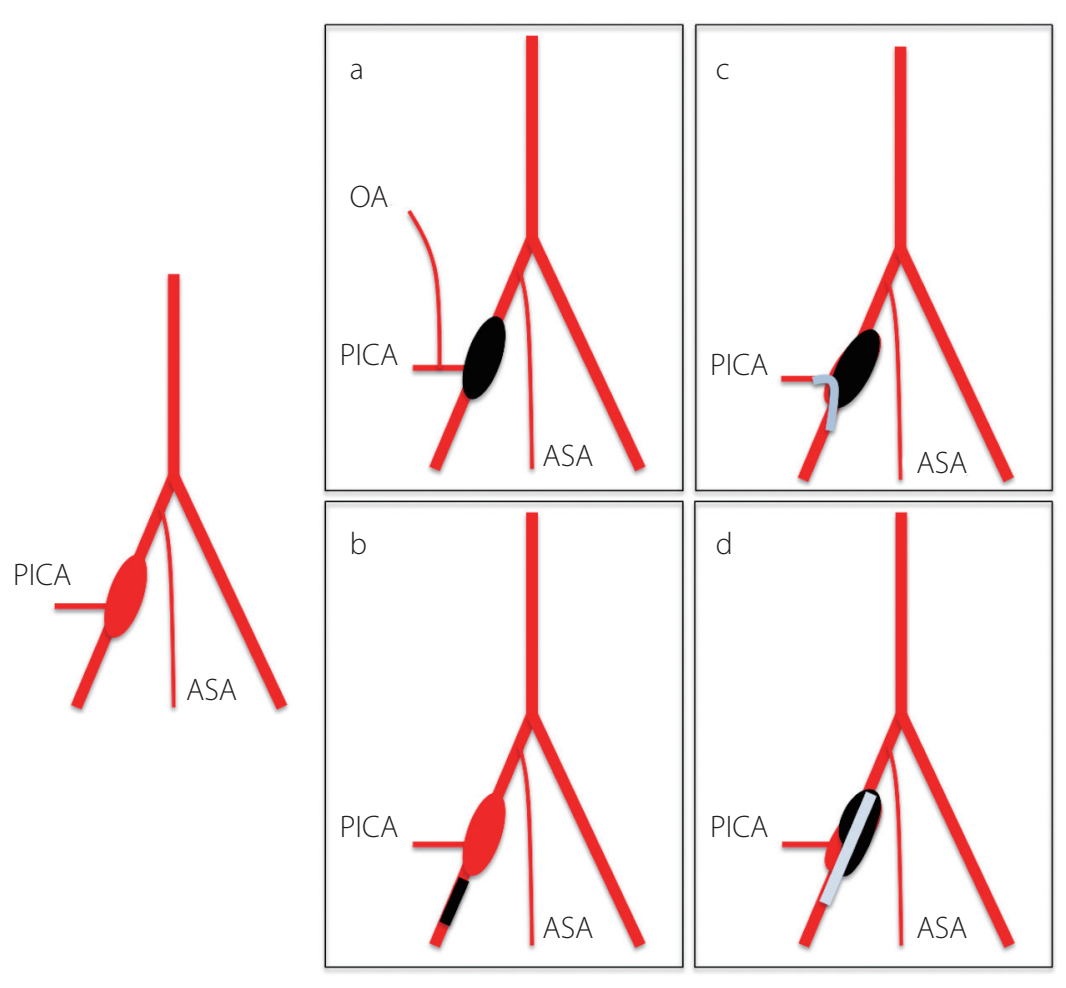

Fig. 2. Schema of the vertebral artery dissecting aneurysm (VADA) treatment. a: vertebral artery (VA) occlusion and occipital artery (OA)-posterior inferior cerebellar artery (PICA) bypass; b: proximal VA trapping to decrease flow to VADA; c: distal VA occlusion and PICA stenting; $\mathrm{d}$ : stenting to the VA (and coiling to VADA). ASA, anterior spinal artery. 
prevent infarction. Previous studies reported infarction or recanalization of the PICA and regrowth of the dissection after endovascular treatment of a VADA., 814,15 The aims of the stent-jack technique for VADA involving the origin of PICA are tight packing of the dissection and PICA preservation. The following issues are associated with this technique. The distal VA needs to be densely embolized and occluded without penetrating the branches of the PICA. After sparsely coiling the proximal VA and entire length of the dissection, stenting from the PICA to the proximal VA is performed. SAC of a VADA may prevent rerupture by densely packing coils into the dilated portion of the dissection, which is a rupture point. However, recurrence has been reported in some cases. ${ }^{16,17}$ A previous study showed recurrence in the proximal segment of the dissection. Therefore, densely packing coils at the entry of the dissection may be important for preventing recurrence. Since indicators of the effectiveness of dense coil packing at the entry of the dissection have not yet been identified, further cases need to be examined.

The stent-jailing technique has been shown to reduce the rate of recurrence more than the stent-jack technique due to fewer mechanical forces and angiogenesis as well as its inhibition of the transforming growth factor $\beta / S$ mad2,3,4 signaling pathway in intracranial aneurysms. ${ }^{18}$ The aneurysm occlusion grade was found to be higher in the stent-jailing group than in the stent-jack group in a 2-year follow-up. In the present study, immediate occlusion was shown, but we did not perform a long-term follow-up. Therefore, long-term monitoring of postoperative changes in a VADA is needed.

Furthermore, additional treatment strategies are required for recanalization by coil transformation just after stenting, such as insertion of the microcatheter distal to the coil mass before stenting, insertion by the transcell technique to the coil mass after stenting, or an approach from the contralateral VA. Moreover, difficulties are associated with estimating whether additional coils protrude into the stent after PICA stenting because PICA is a tortuous artery and it is technically challenging to take the down-the-barrel view position in order to identify the lumen of the PICA. We need to consider a coping strategy, such as balloon inflation into the stent, to prevent coil protrusion and overlap stenting when coil protrusion occurs. ${ }^{19}$ Enterprise VRD is effective for the stent-jack technique on the PICA because we may perform additional coiling using the transcell technique and the stent may compress the coil due to the strong radial forces of an Enterprise VRD. In cases in which a PICA arises from the VA at an acute angle, antegrade stenting is not required and we need to consider retrograde stenting from the contralateral VA to PICA. Therefore, several alternative strategies need to be planned when endovascular treatments are performed for VADA involving the origin of PICA.

\section{CONCLUSION}

The stent-jack technique for a ruptured vertebral artery dissecting aneurysm involving the origin of the posterior inferior cerebellar artery is effective. However, careful attention to recanalization after stenting is needed due to transformation of the coil mass.

\section{Fund}

None.

\section{Ethics Statement}

This type of study does not require approval from the IRB.

\section{Conflicts of Interest}

The authors have no conflicts to disclose.

\section{Author Contribution}

Concept and design: TT and YN. Writing the article: TT. Critical revision of the article: $\mathrm{YN}, \mathrm{Gl}, \mathrm{KT}, \mathrm{SH}, \mathrm{KA}, \mathrm{HI}, \mathrm{TK}, \mathrm{MS}$, and KU. Final approval of the article: KU. Obtained funding: none. Overall responsibility: $\mathrm{TT}$ and $\mathrm{YN}$.

\section{ORCID}

Toshitsugu Terakado: https://orcid.org/0000-0001-9056-1220 Yasunobu Nakai: https://orcid.org/0000-0002-9956-2075 Go Ikeda: https://orcid.org/0000-0002-4373-9193 Sho Hanai: https://orcid.org/0000-0002-5318-3715 Kazuki Akutagawa: https://orcid.org/0000-0003-0462-7073 Takahiro Konishi: https://orcid.org/0000-0002-9845-4048 Masanari Shiigai: https://orcid.org/0000-0003-4227-7334 Kazuya Uemura: https://orcid.org/0000-0003-0211-1153

\section{REFERENCES}

1. de Paula Lucas C, Piotin M, Spelle L, Moret J. Stent-jack technique in stent-assisted coiling of wide-neck aneurysms. Neurosurgery 2008;62(5 Suppl 2):ONS414-416; discussion ONS416-417 
2. Yamada M, Kitahara T, Kurata A, Fujii K, Miyasaka Y. Intracranial vertebral artery dissection with subarachnoid hemorrhage: clinical characteristics and outcomes in conservatively treated patients. J Neurosurg 2004;101:25-30

3. Mizutani T, Aruga T, Kirino T, Miki Y, Saito I, Tsuchida T. Recurrent subarachnoid hemorrhage from untreated ruptured vertebrobasilar dissecting aneurysms. Neurosurgery 1995;36:905-911; discussion 912-913

4. Guan J, Li G, Kong X, He C, Long J, Qin H, et al. Endovascular treatment for ruptured and unruptured vertebral artery dissecting aneurysms: a meta-analysis. J Neurointerv Surg 2017;9:558563

5. Ahn JY, Han IB, Kim TG, Yoon PH, Lee YJ, Lee BH, et al. Endovascular treatment of intracranial vertebral artery dissections with stent placement or stent-assisted coiling. AJNR Am J Neuroradiol 2006;27:1514-1520

6. Satow T, Ishii D, lihara K, Sakai N; JR-NET study group. Endovascular treatment for ruptured vertebral artery dissecting aneurysms: results from Japanese Registry of Neuroendovascular Therapy (JR-NET) 1 and 2. Neurol Med Chir (Tokyo) 2014;54:98106

7. lihara K, Sakai N, Murao K, Sakai H, Higashi T, Kogure S, et al. Dissecting aneurysms of the vertebral artery: a management strategy. J Neurosurg 2002;97:259-267

8. Cho DY, Choi JH, Kim BS, Shin YS. Comparison of clinical and radiologic outcomes of diverse endovascular treatments in vertebral artery dissecting aneurysm involving the origin of PICA. World Neurosurg 2019;121:e22-e31

9. Nakamura H, Fujinaka T, Nishida T, Kishima H, Sakai N; JR-NET3 study group. Endovascular therapy for ruptured vertebral artery dissecting aneurysms: results from nationwide, retrospective, multi-center registries in Japan (JR-NET3). Neurol Med Chir (Tokyo) 2019;59:10-18

10. Chen JA, Garrett MC, Mlikotic A, Ausman Jl. Treatment of intracranial vertebral artery dissecting aneurysms involving the posterior inferior cerebellar artery origin. Surg Neurol Int 2019;10:116
11. Ryu CW, Park S, Shin HS, Koh JS. Complications in stent-assisted endovascular therapy of ruptured intracranial aneurysms and relevance to antiplatelet administration: a systematic review. AJNR Am J Neuroradiol 2015;36:1682-1688

12. Turk AS, Ahmed A, Niemann DB, Aagaard-Kienitz B, Brooks N, Levine RL. Utilization of self-expanding stents in the treatment of intracranial atherosclerotic disease in the distal small cerebral vessels. Neuroradiology 2007;49:659-663

13. Siddiqui MA, J Bhattacharya J, Lindsay KW, Jenkins S. Horizontal stent-assisted coil embolisation of wide-necked intracranial aneurysms with the Enterprise stent--a case series with early angiographic follow-up. Neuroradiology 2009;51:411-418

14. Chung J, Kim BS, Lee D, Kim TH, Shin YS. Vertebral artery occlusion with vertebral artery-to-posterior inferior cerebellar artery stenting for preservation of the PICA in treating ruptured vertebral artery dissection. Acta Neurochir (Wien) 2010;152:14891492

15. Ota T, Sato M, Amano T, Saito A, Matsumaru Y. Reconstructive endovascular treatment of ruptured vertebral artery dissection involving the posterior inferior cerebellar artery. Acta Neurochir (Wien) 2016;158:1089-1093

16. Phatouros CC, Sasaki TY, Higashida RT, Malek AM, Meyers PM, Dowd CF, et al. Stent-supported coil embolization: the treatment of fusiform and wide-neck aneurysms and pseudoaneurysms. Neurosurgery 2000;47:107-113; discussion 113-115

17. Kim BM, Shin YS, Kim SH, Suh SH, Ihn YK, Kim DI, et al. Incidence and risk factors of recurrence after endovascular treatment of intracranial vertebrobasilar dissecting aneurysms. Stroke 2011;42:2425-2430

18. Xu N, Meng H, Liu T, Feng Y, Qi Y, Zhang D, et al. Stent-jailing technique reduces aneurysm recurrence more than stent-jack technique by causing less mechanical forces and angiogenesis and inhibiting TGF- $\beta / S m a d 2,3,4$ signaling pathway in intracranial aneurysm patients. Front Physio/ 2019;9:1862

19. Kim BM, Kim DJ, Kim DI. Stent application for the treatment of cerebral aneurysms. Neurointervention 2011;6:53-70 